\title{
Experimental verification of acoustic pseudospin multipoles in a symmetry-broken snowflakelike topological insulator
}

\author{
Zhiwang Zhang, ${ }^{1}$ Ye Tian, ${ }^{1}$ Ying Cheng,,${ }^{1, *}$ Xiaojun Liu, ${ }^{1, \dagger}$ and Johan Christensen ${ }^{2, \ddagger}$ \\ ${ }^{1}$ Department of Physics, Collaborative Innovation Center of Advanced Microstructures, Nanjing University, Nanjing 210093, China \\ ${ }^{2}$ Instituto Gregorio Millan Barbany, Universidad Carlos III de Madrid, ES-28916 Leganés, Madrid, Spain
}

(Received 23 October 2017; published 29 December 2017)

\begin{abstract}
Topologically protected wave engineering in artificially structured media resides at the frontier of ongoing metamaterials research, which is inspired by quantum mechanics. Acoustic analogs of electronic topological insulators have recently led to a wealth of new opportunities in manipulating sound propagation by means of robust edge mode excitations through analogies drawn to exotic quantum states. A variety of artificial acoustic systems hosting topological edge states have been proposed analogous to the quantum Hall effect, topological insulators, and Floquet topological insulators in electronic systems. However, those systems were characterized by a fixed geometry and a very narrow frequency response, which severely hinders the exploration and design of useful applications. Here we establish acoustic multipolar pseudospin states as an engineering degree of freedom in time-reversal invariant flow-free phononic crystals and develop reconfigurable topological insulators through rotation of their meta-atoms and reshaping of the metamolecules. Specifically, we show how rotation forms man-made snowflakelike molecules, whose topological phase mimics pseudospin-down (pseudospin-up) dipolar and quadrupolar states, which are responsible for a plethora of robust edge confined properties and topological controlled refraction disobeying Snell's law.
\end{abstract}

DOI: 10.1103/PhysRevB.96.241306

Topology is a mathematical concept, which describes the properties of space that are preserved under continuous deformations. Topological states have also been extended to condensed-matter physics based on the quantum Hall effect (QHE) [1,2], the quantum spin Hall effect (QSHE) [3,4], and topological insulators (TIs) [5,6]. Over the past ten years, investigation into new topologically protected edge states has started to grow in other subfields of physics, such as photonics [7-16], phononics [17-32], and mechanics [33-36]. The intrinsic difference between electrons and acoustic waves represents a great challenge in creating the spinlike degree of freedom for sound only possessing longitudinal polarization. To resolve this obstacle, analogous unidirectional edge channels have been demonstrated in phononic crystals (PnC), which were constructed with circulating flow fields [18-21,37] to break the time-reversal symmetry to mimic the QHE. Similarly, coupled ring resonator waveguides were proposed analogous to a Floquet insulator [23-25]. Beyond that, valley-projected acoustic topological insulators were proposed to obtain backscattering-immune valley transport [30,32]. However, the inherent losses and noise that intrinsically accompany acoustic propagation in moving media, together with considerable fabrication complexities of ring waveguides may become detrimental in future topological applications. Most recently, phononic "graphene" with double Dirac cones [27-29] has been proposed for the design of two-dimensional (2D) acoustic $T$-symmetric TIs, functioning without applying external "forces." The band inversion between pseudospin states has been induced by varying the radius of the constitutive meta-atoms. This approach, however, is prone to imperfections as to the exact radii and site of these atoms, making the

\footnotetext{
*chengying@nju.edu.cn

†liuxiaojun@nju.edu.cn

†johan.christensen@uc3m.es
}

reconfiguration and material processing between trivial and nontrivial topological regions a great technical challenge. Also, the lack of tunability and adaptation to functional needs and robust applications requires an eased strategy toward reconfigurable TIs in artificially structured media.

Based on inherently time-reversal invariant constituents, in this Rapid Communication, we propose to open nontrivial band gaps in order to produce edge states by lifting the degeneracy associated with double Dirac cones ([38], Note I). We introduce the mirror-symmetry-breaking mechanism $[32,39,40]$ to construct a reconfigurable topological insulator. To accomplish this, anisotropic scatterers are used to close and reopen the band gap. The chirality of the acoustic vortices surrounding the metamolecules (i.e., right- or lefthand circular polarized, labeled as RCP and LCP) plays the role of the pseudospin degree of freedom in the 2D snowflakelike TI. Simply by rotating the rods, one is able to reconfigure the metamolecules to generate the topological transition from trivial to nontrivial via pseudospin dipoles and quadrupoles. We also demonstrate that an interface between trivial and nontrivial TIs supports highly confined topologically protected edge states, which are not vulnerable to defects. Aside from the highly flexible approach to acquire topological phases through the rotation of the involved scatterers, we also display how this scheme can lead to topologically controlled unusual refraction, both with negative angles and unexpected birefringence.

The intrinsic difference between spin-1/2 electron and spin-1 photon/phonon is the Kramers doublet, which is the prerequisite condition for the QSHE, nonexistent in acoustic systems. However, to construct an artificial acoustic Kramers doublet, the degree of freedom must be increased to obtain a pair of pseudospin states analogous to electrons [27,28]. As a result, fourfold-degenerate double Dirac cones in acoustic band structure are required, which can be engineered in a triangular/honeycomb lattice $[41,42]$. In order to introduce the mirror-symmetry-breaking mechanism, we construct 
(a)

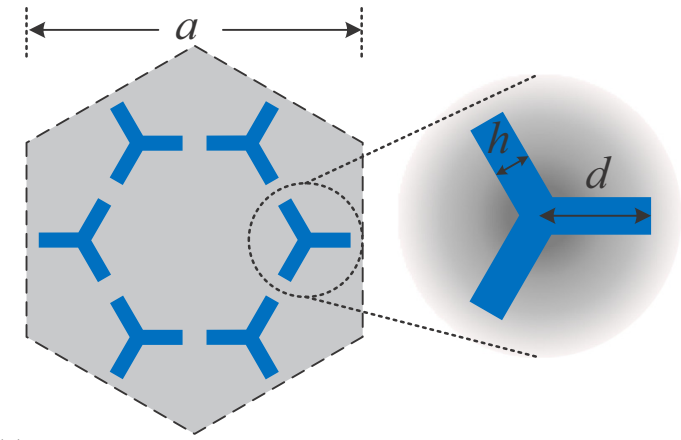

(c)

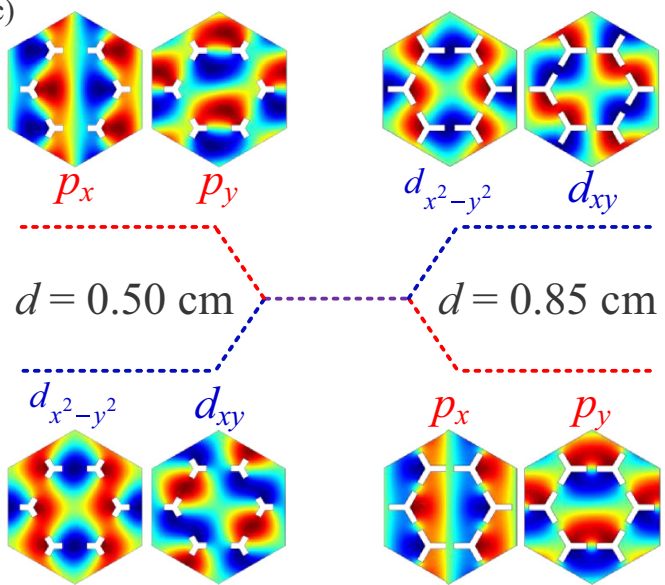

(b)

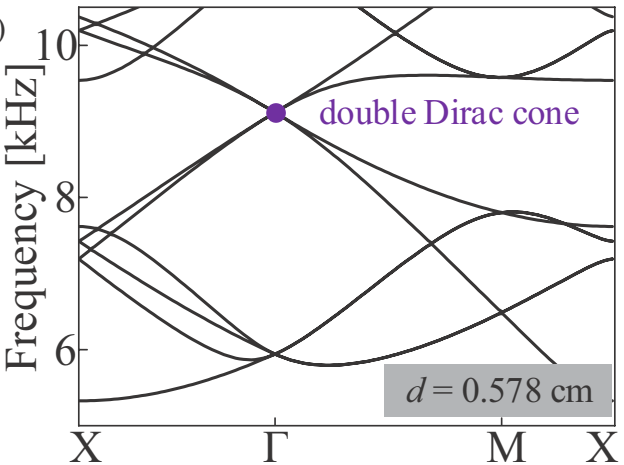

(d)

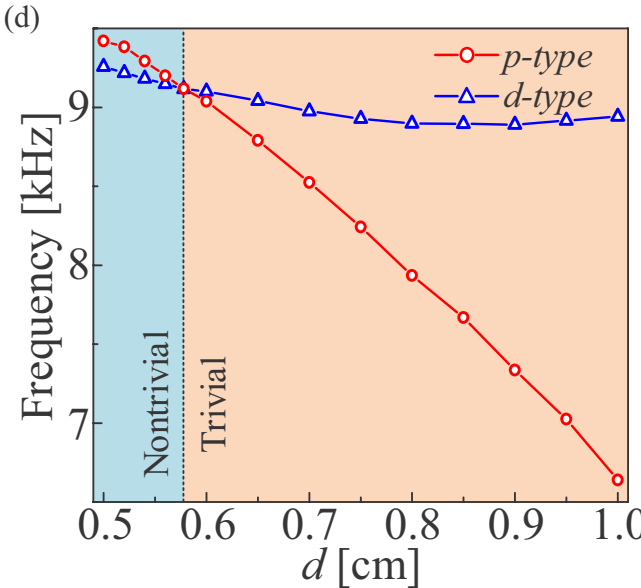

FIG. 1. (a) Schematic of the snowflakelike metamolecule unit of the phononic triangular lattice composed of TLRs. (b) Dispersion relation of the structure in which $d=0.578 \mathrm{~cm}$. (c) Topological modes inversion underlying the transition of pseudospin states. The rainbow color represents the amplitude of total pressure fields. $p_{x}$ and $p_{y}$ show pseudospin dipole modes, while $d_{x y}$ and $d_{x^{2}-y^{2}}$ show pseudospin quadrupole modes. (d) The eigenfrequencies of the double twofold degenerated states are controlled by the length of the arm $d$ at the BZ center. The band inversion effect is clearly observed as indicated in different colors.

three-legged rods (TLRs) made of epoxy resin, in which the $C_{6 v}$ symmetry is invariable as shown in Fig. 1(a). In the following, we design "snowflake" metamolecules containing six neighboring TLRs as the primitive cells, which form the triangular lattice [see Fig. 1(a)]. The distance between snowflake metamolecules, which are embedded into air, is $a=6.51 \mathrm{~cm}$ and the width of the legs is $h=0.30 \mathrm{~cm}$, which we hold constant. The filling factor is manipulated through changing the length of the legs $d$. With these geometrical parameters and choosing $d=0.578 \mathrm{~cm}$, we depict in Fig. 1(b) how the double Dirac cone is formed. By varying the filling fraction such that $d=0.85 \mathrm{~cm}$, a double twofold degeneracy, one for the lower bands of the $p$ type and the other for the upper bands of the $d$ type, appears at the Brillouin zone (BZ) center, as shown in Fig. 1(c) [28]. Interestingly, a pair of dipolar resonance states are accompanied by a pair of quadrupolar resonance states: similar to $p$ and $d$ orbitals of electrons, here the dipoles are even or odd symmetrical to the axes $x / y$ (corresponding to $p_{x} / p_{y}$ ), while the quadrupole is odd symmetrical to the axes $x$ and $y$ (corresponding to $d_{x y}$ ), or even symmetrical to the axes $x$ and $y$ at the same time (corresponding to $d_{x^{2}-y^{2}}$ ). With dependence to the length $d$, Fig. 1(d) shows the band inversion process between $d$-type and p-type states, which illustrates the process in which the topological band gap is closed and reopened by changing the filling factor enabling trivial and nontrivial states. But during this process, there are two points that have to be emphasized: (1) The filling ratio is limited in the range from 0.5 to $1.0 \mathrm{~cm}$ because the conelike dispersion will disappear when the filling ratio is beyond the range. (2) The frequency range of nontrivial states is very narrow and has no intersection with the trivial states as can be clearly seen in Fig. 1(d). This disadvantage restricts the exploration of realistic applications in acoustic topological insulators.

To counteract this problem and being able to construct reconfigurable TIs, we demonstrate that a simple rotation of the TLRs can pose enhanced control over topological phases ([38], Note II). We begin by designing the snowflake metamolecule with a constant leg length of $d=0.85 \mathrm{~cm}$. As shown in Fig. 2(a) we denote this system the type- $A$ configuration. By rotating the TLRs, we adjust the structure into a new metamolecule that constitutes the type- $B$ configuration, in which the mirror symmetry is broken as shown in Fig. 2(b). As seen in Figs. 2(c) and 2(d), a notably broad band gap is formed that will prove useful as we will show later in the experiments. The dispersion relations for these two structures are calculated using COMSOL MULTIPHYSICS, a finite-element solver software ([38], Note III). The fourfold-degenerate states split into two twofold-degenerate states and a total band gap is obtained in both situations. Plotted in the insets 
(a)

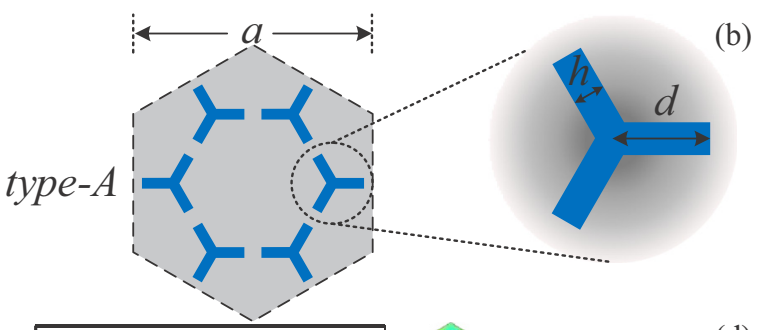

(c)

10

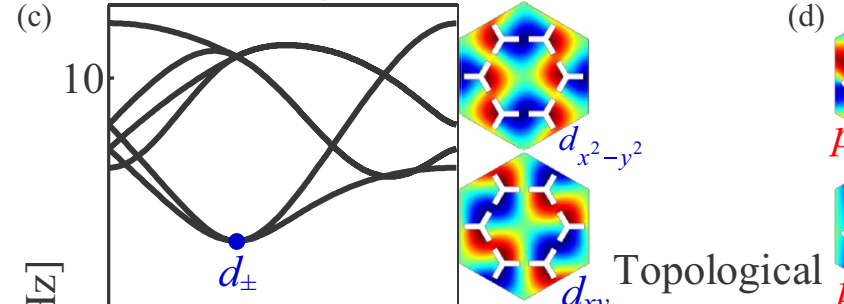

4

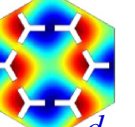

)

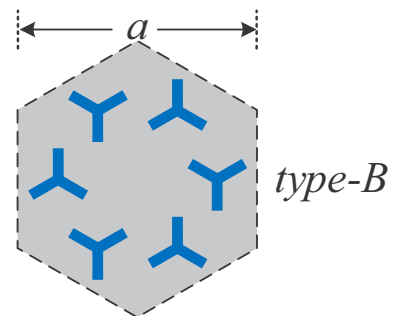

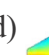

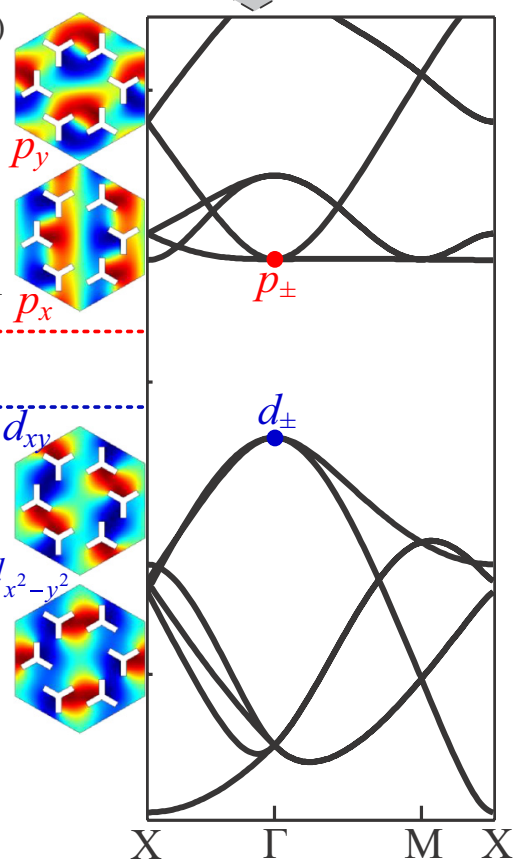

(e)

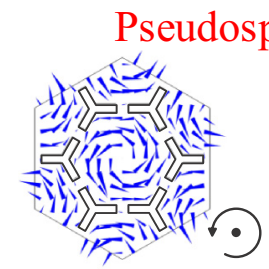

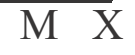

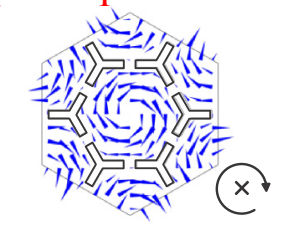

(f) Pseudospin Quadrupole
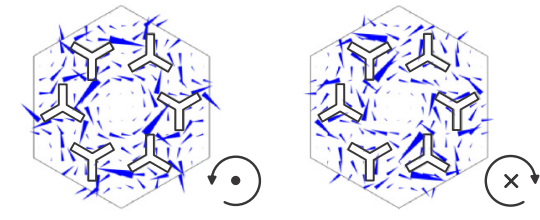

FIG. 2. Schematics of two snowflakelike metamolecules: the topologically (a) trivial and (b) nontrivial triangular lattice composed of TLRs with $d=0.85 \mathrm{~cm}$. Corresponding dispersion relations of the topologically (c) trivial regime and (d) nontrivial structure. Insets: topological modes inversion underlying the transition between pseudospin states. Sound intensity $\boldsymbol{I}$ associated with pseudospin-down and pseudospin-up modes at the $\Gamma$ point below the band gap for the (e) trivial and (f) nontrivial regimes. The arrows show the direction and amplitude of the intensity.

of Figs. 2(c) and 2(d), we show the distributions of the pressure fields at the BZ, spectrally corresponding to the colored dots at the $\Gamma$ point. We demonstrate that similar dipolar resonance states, accompanied by a pair of quadrupolar resonance states, are created as compared with Fig. 1(c). The angular momenta of the wave function of pressure fields $p_{ \pm}=\left(p_{x} \pm i p_{y}\right) / \sqrt{2}$ and $d_{ \pm}=\left(d_{x^{2}-y^{2}} \pm i d_{x y}\right) / \sqrt{2}$ further constitute the spin-1/2 for the bulk states (pseudospin) in the present acoustic crystals based on snowflake metamolecules. To deliberately observe the pseudospin states, we examine the real-space distributions of the root-mean-square intensity $\boldsymbol{I}=\frac{1}{T} \int_{0}^{T} \operatorname{Re}(\mathrm{p}) \operatorname{Re}(\boldsymbol{v}) d t$ with $T=\frac{2 \pi}{\omega}$, which expresses the energy flow in the metamolecule. For simplicity, only the states below the band gap are illustrated in Figs. 2(e) and 2(f). The time-dependent spinning of intensity around the
$\mathrm{BZ}$ center with its respective $\mathrm{RCP} / \mathrm{LCP}$ chirality, is clearly visualized in a movie ([38], movie 1). We underline that the energy vortex, in the absence of a background fluid flow, is resonance based and that its chirality corresponds to the pseudospin-down and pseudospin-up states as has been unequivocally demonstrated. Instead of refabricating the entire crystal structure, we presented how band inversion takes place under the rotation of the TLRs through symmetry breaking while keeping the lattice intact. For the type- $A$ configuration shown in Fig. 2(c), two twofold degeneracies are created, one for the lower bands $p_{x} / p_{y}$ (pseudospin dipoles) and the other for the upper bands $d_{x y} / d_{x^{2}-y^{2}}$ (pseudospin quadrupoles). However, for type- $B$ configuration shown in Fig. 2(d), the dipole states rise above the quadrupole states, leading to an inverted band structure. The proposed band inversion further 
leads to a topological transition from a topologically trivial state to a nontrivial state, to which we numerically calculate the $\mathbb{Z}_{2}$ topological invariant using a $\boldsymbol{k} \cdot \boldsymbol{P}$ perturbation method for verification. The effective Hamiltonian, in the vicinity of the $\Gamma$ point can be simplified by the following matrix:

$$
H^{\mathrm{eff}}(\boldsymbol{k})=\left(\begin{array}{cccc}
M-B k^{2} & A k_{+} & 0 & 0 \\
A^{*} k_{-} & -M+B k^{2} & 0 & 0 \\
0 & 0 & M-B k^{2} & A k_{-} \\
0 & 0 & A^{*} k_{+} & -M+B k^{2}
\end{array}\right),
$$

where $k_{ \pm}=k_{x} \pm i k_{y}$ and $M=\left(\varepsilon_{d}-\varepsilon_{p}\right) / 2$ is the frequency difference between dipolar and quadrupolar pseudospin eigen- states, which is positive for the trivial and negative for the nontrivial case. $A$ is determined by the off-diagonal elements of the first-order perturbation term. $B$ is found from the diagonal elements of the second-order perturbation term, and is typically negative. On the basis of Eq. (1), the Chern numbers can be evaluated as $[4,43]$

$$
C_{ \pm}= \pm \frac{1}{2}[\operatorname{sgn}(M)+\operatorname{sgn}(B)] .
$$

For the type- $A$ configuration in Fig. 2(c), we obtain the Chern number $C_{ \pm}=0$ where $M=\left(\varepsilon_{d}-\varepsilon_{p}\right) / 2>0$, indicating that the band gap is trivial. Contrary to this, for the type- $B$ configuration in Fig. $2(\mathrm{~d}), M=\left(\varepsilon_{d}-\varepsilon_{p}\right) / 2<0$ with the $p$-like states spectrally exceeding the $d$-like states, we
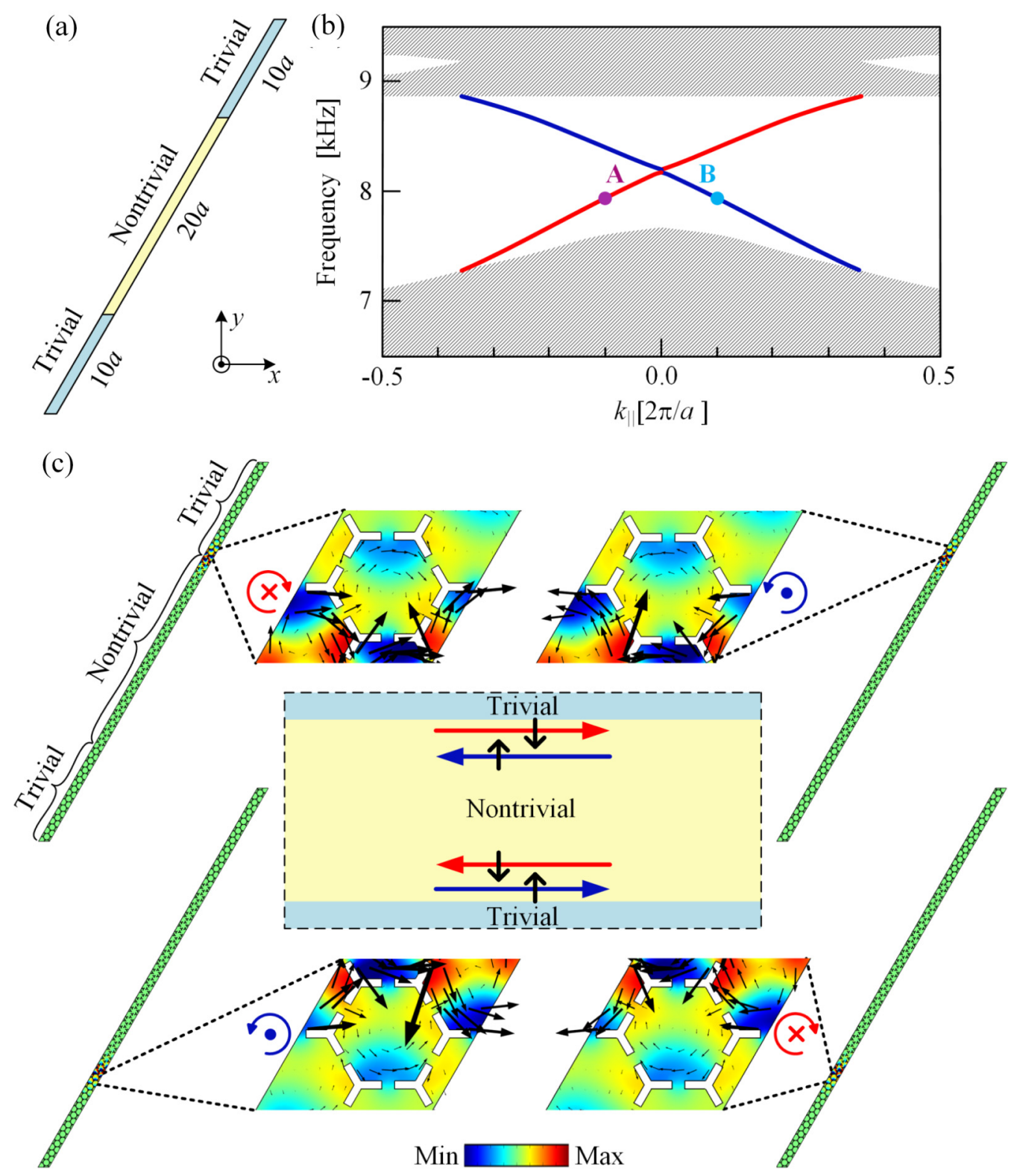

FIG. 3. (a) Schematic of a ribbon-shaped supercell composed of 20 for the topologically nontrivial region and two times ten metamolecules for the trivial regions, respectively. (b) Dispersion relation of the ribbon-shaped supercell. Dark-gray regions indicate the bulk states, whereas the doubly degenerated solid red and blue lines represent the edge states of opposite group velocities. (c) Acoustic pressure fields and intensity of the pseudospin-dependent one-way edge modes localized at the interface of the supercell, corresponding to the points $A$ and $B$ indicated in (b). 

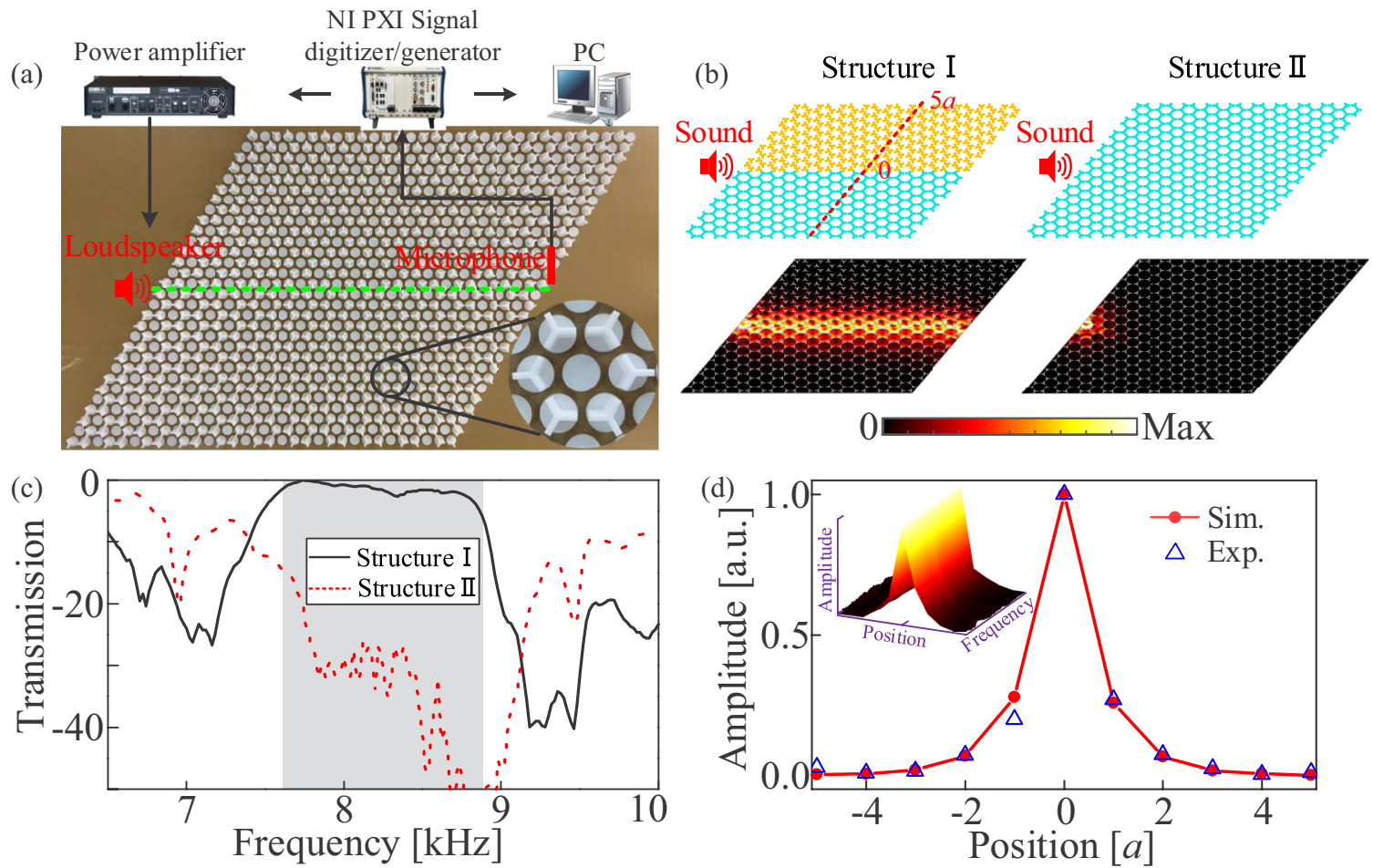

FIG. 4. (a) Photograph of a fabricated PnC sample (without the top cover). (b) Simulated distributions of the absolute pressure fields along the interfaces between trivial and nontrivial regions (left panel, structure I) and through the trivial bulk region (right panel, structure II). Cyan and yellow regions represent the trivial and nontrivial regimes. (c) Experimentally measured transmission spectra of topological edge states and bulk states. (d) Frequency-dependent spatial profiles of the absolute pressure fields measured in the middle of the crystal [red dashed line in (b)]. The frequency range corresponds to the one in (c).

obtain a Chern number $C_{ \pm}= \pm 1$, which indicates the band gap is nontrivial. To recap, we have confirmed a topological phase transition from a trivial (type- $A$ ) to a nontrivial (type- $B$ ) state via band inversion, induced by simply rotating the TLRs.

According to the bulk-boundary correspondence [44], there will be unidirectional acoustic edge states at the interface between a trivial and nontrivial system, analogous to the QSHE in electronics. To confirm the existence of these topologically protected edge states, we numerically calculate the band structure for a ribbon-shaped supercell, as shown in Figs. 3(a) and $3(\mathrm{~b})$. The frequency range of the topological band gap spans from 7653 to $8859 \mathrm{~Hz}$. The real-space distributions of the pressure and intensity fields at a typical value of the momentum $k_{\|}= \pm 0.1 \times 2 \pi / a$, corresponding to points $A$ and $B$ in Fig. 3(b), are illustrated in Fig. 3(c), which is very similar to the acoustic pseudospin modes from Fig. 2 . Here we illustrate, with corresponding edge mode chirality, how these states confine along the two interfaces of the snowflake ribbon: the upper edge contains a forward channel with pseudospindown and a backward channel with pseudospin-up, whereas this behavior is entirely reverse at the lower edge.

We construct a topologically protected waveguide (TPWG) in a finite $10 a \times 10 a$ lattice as shown in Fig. 4(a) to experimentally verify the reflection-free transmission of topological edge states ([38], Sec. IV). The simulated pressure fields at frequency $f=8200 \mathrm{~Hz}$, which is in the topological band gap, are illustrated in Fig. 4(b). Because of the topological protection of edge states at the interface between the trivial and nontrivial system, the sound waves transmit along the interface without backscattering for structure I as opposed to structure II, the trivial system alone, in which pure isolation occurs. The experimental data shown in Fig 4(c) illustrate the measured $\sim 30 \mathrm{~dB}$ transmission enhancement of edge state (black solid line) as compared to the bulk state (red dashed line). In Fig. 4(d), we simulate and experimentally measure the amplitude of the pressure field at frequency $f=8200 \mathrm{~Hz}$ across the crystal array as is indicated by the red dashed line in Fig. 4(b). The experimental results are in good agreement with the simulated ones and show that sound transmission along TPWG exponentially decays into the bulk, which is one of the key characteristics of TPWG. In the inset of Fig. 4(d), we emphasize how the tightness of the confined edge modes prevails over an extended frequency range corresponding to the width of the topological band gap ([38], Sec. V).

Next, we experimentally observe the most important physical property of TIs: the topological robustness against defects. We introduce a number of sharp bends into the TPWG as shown in Fig. 5(a). We also construct a topologically trivial waveguide (TTWG) by simply removing a row of TLRs. Figures 5(a) and 5(b) show pressure fields within the TPWG and the TTWG inside the band gap. The experimental transmission spectra are shown in Figs. 5(c) and 5(d). Notice that the TPWG clearly sustains strong robustness against the introduced structural bends as seen in Figs. 5(a) and 5(c), where the transmittance remains broad and high, which leads to a robust power flow along the bends. Due to the lack of topological protection of the TPWG, insertion of sharp bends into this system leads to 

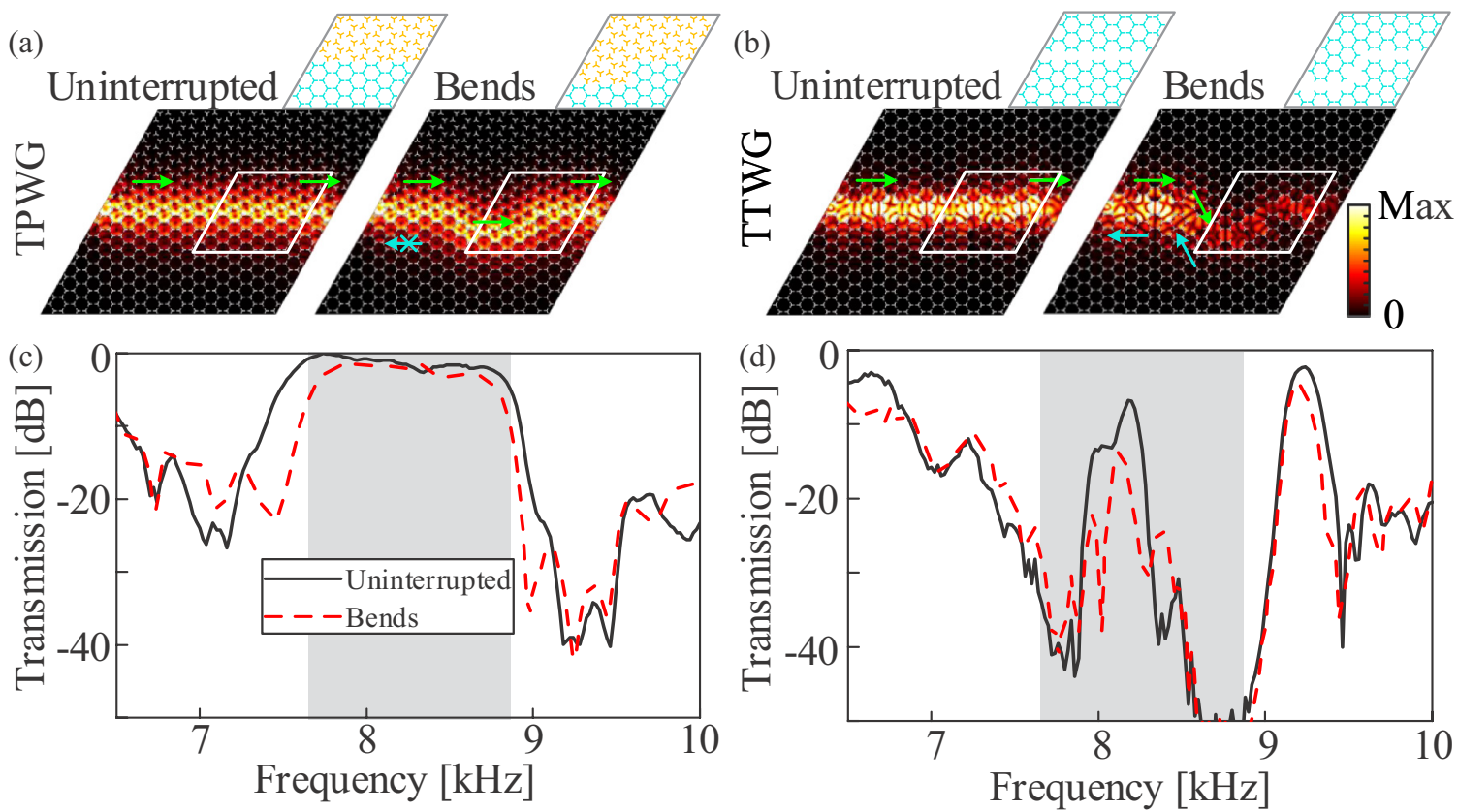

FIG. 5. Simulated absolute pressure fields (a) at the frequency $f=8200 \mathrm{~Hz}$ in the TPWG and (b) at the frequency of $f=8000 \mathrm{~Hz}$ in the TTWG. Green and cyan arrows denote the directions of transmitted and reflected sound waves, respectively. Inset: the partial structures of the uninterrupted interface and the interfaces with bends. (c)-(d) Experimental transmission spectra for the TPWG and the TTWG, respectively. Shaded regions represent the band gap.

strong backscattering of edge states and significant reduction of the output transmittance as seen in Figs. 5(b) and 5(d).

Finally, we explore the possibility to design man-made acoustic refraction as controlled by the topology in the PnC. First, as depicted in Figs. 6(a)-6(c), we launch sound waves at the edge of two TPWGs of exact opposite configuration. Due to the degenerate pseudospin states, the outgoing wave converts into two beams. It is noted that the topological birefringence, despite some intensity differences, seem not to be affected by the placement of the trivial/nontrivial regions (a)

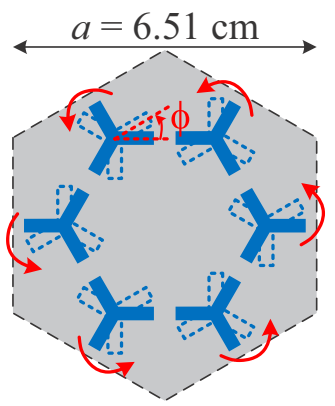

(d)

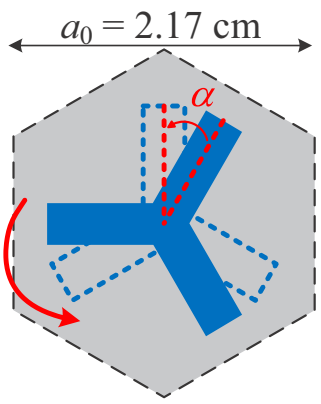

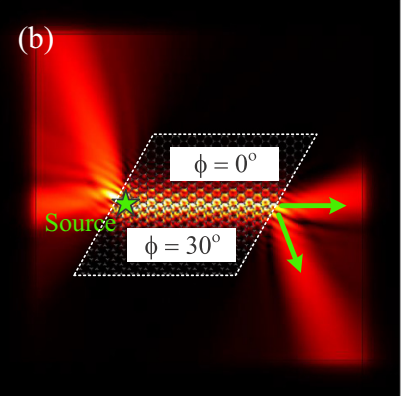
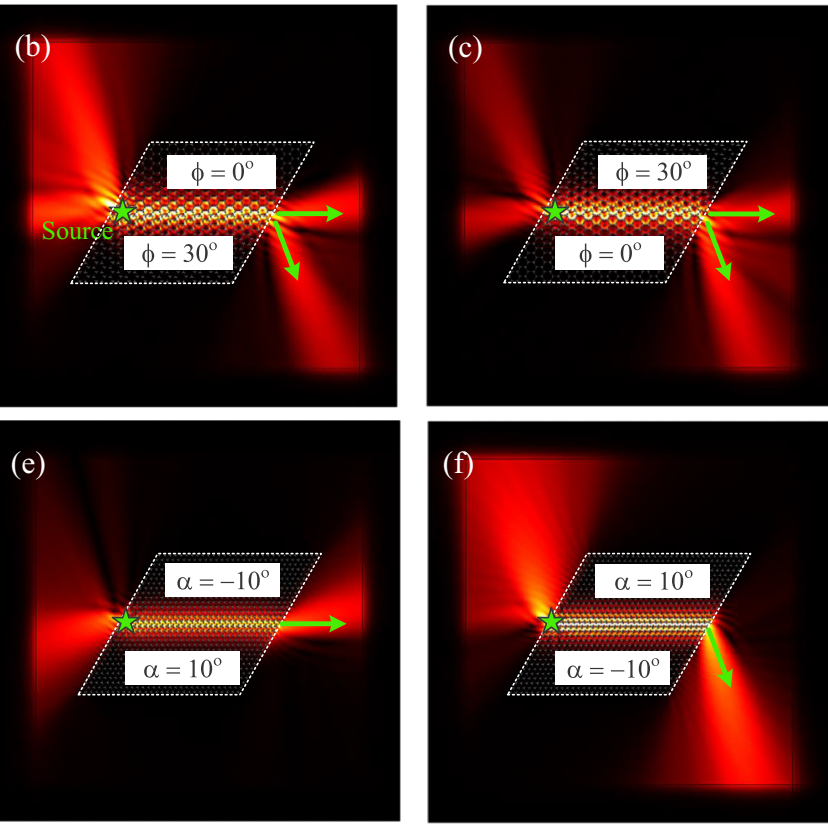

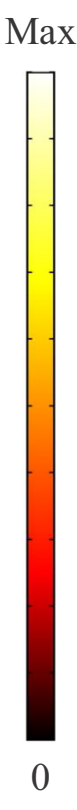

FIG. 6. (a) Schematic of the metamolecule with the rotation angle $\phi$, which is positive/negative when rotating TLRs anticlockwise/clockwise. Topological birefringence: pressure fields along the interface with (b) $\phi=0^{\circ}$ (trivial) above and $\phi=30^{\circ}$ (nontrivial) below; (c) $\phi=30^{\circ}$ (nontrivial) above and $\phi=0^{\circ}$ (trivial) below. (d) Schematic of primitive cell of triangular lattice with lattice constant $a_{0}=2.17 \mathrm{~cm}$. Topological positive/negative refraction: pressure fields along the interface with (b) $\alpha=-10^{\circ}$ above and $\alpha=10^{\circ}$ below; (c) $\alpha=10^{\circ}$ above and $\alpha=-10^{\circ}$ below. 
as measured by $\phi$. This scenario is entirely different if we introduce a single TLR into the unit cell [Fig. 6(d)]. This modification supports a single polarization only as compared to the snowflake metamolecule. Hence, the result of varying $\alpha$ as depicted in Figs. 6(d)-6(f), displays that we are able, in reference to the PnC crystal termination, to efficiently launch a collimated beam of either positive or negative refraction. These results prove that both unusual birefringence and negative refraction can be engineered through the topology in acoustic lattices. Conventionally, in the field of acoustic communications, coupling between waveguides and free space is a challenging problem to overcome based on the inherent impedance mismatch. Not only can refraction be engineered at will, but we show that topological waveguides provide a robust platform to smoothly outcouple sound into free space without any reflection as shown in Fig. 6.

To conclude, we have implemented a 2D acoustic topological insulator analogous to QSHE in electronic systems, whose topological phase can be reconfigured by simply rotating the meta-atoms within the unit cells. The handedness of the acoustic vortices plays the role of the pseudospin (pseudospin-down or pseudospin-up) via their multipolar order. The topological properties proposed in this work can be useful for one-way signal processing regardless of structural defects, which will be highly useful for noise control and engineered acoustic refraction.

This work was supported by National Key R\&D Program of China (2017YFA0303702), NSFC (Grants No. 11674172, No. 11574148, No. 11474162), Jiangsu Provincial NSF (BK20160018), and Postgraduate Research \& Practice Innovation Program of Jiangsu Province (KYCX17 0020). J.C. acknowledges the support from the European Research Council (ERC) through the Starting Grant 714577 PHONOMETA and from the MINECO through a Ramón y Cajal grant (Grant No. RYC-2015-17156).
[1] R. B. Laughlin, Phys. Rev. Lett. 50, 1395 (1983).

[2] K. V. Klitzing, G. Dorda, and M. Pepper, Phys. Rev. Lett. 45, 494 (1980).

[3] C. L. Kane and E. J. Mele, Phys. Rev. Lett. 95, 226801 (2005).

[4] B. A. Bernevig, T. L. Hughes, and S.-C. Zhang, Science 314, 1757 (2006).

[5] M. Z. Hasan and C. L. Kane, Rev. Mod. Phys. 82, 3045 (2010).

[6] X.-L. Qi and S.-C. Zhang, Rev. Mod. Phys. 83, 1057 (2011).

[7] W. Gao, M. Lawrence, B. Yang, F. Liu, F. Fang, B. Béri, J. Li, and S. Zhang, Phys. Rev. Lett. 114, 037402 (2015).

[8] F. D. M. Haldane and S. Raghu, Phys. Rev. Lett. 100, 013904 (2008).

[9] Z. Wang, Y. Chong, J. D. Joannopoulos, and M. Soljacic, Nature (London) 461, 772 (2009).

[10] K. Fang, Z. Yu, and S. Fan, Nat. Photonics 6, 782 (2012).

[11] A. B. Khanikaev, S. Hossein Mousavi, W.-K. Tse, M. Kargarian, A. H. MacDonald, and G. Shvets, Nat. Mater. 12, 233 (2013).

[12] T. Ma, A. B. Khanikaev, S. H. Mousavi, and G. Shvets, Phys. Rev. Lett. 114, 127401 (2015).

[13] G. Q. Liang and Y. D. Chong, Phys. Rev. Lett. 110, 203904 (2013).

[14] M. Hafezi, E. A. Demler, M. D. Lukin, and J. M. Taylor, Nat. Phys. 7, 907 (2011).

[15] M. Hafezi, S. Mittal, J. Fan, A. Migdall, and J. M. Taylor, Nat. Photonics 7, 1001 (2013).

[16] L.-H. Wu and X. Hu, Phys. Rev. Lett. 114, 223901 (2015).

[17] V. Peano, C. Brendel, M. Schmidt, and F. Marquardt, Phys. Rev. X 5, 031011 (2015).

[18] A. B. Khanikaev, R. Fleury, S. H. Mousavi, and A. Alu, Nat. Commun. 6, 8260 (2015).

[19] Z. Yang, F. Gao, X. Shi, X. Lin, Z. Gao, Y. Chong, and B. Zhang, Phys. Rev. Lett. 114, 114301 (2015).

[20] X. Ni, C. He, X. C. Sun, X. P. Liu, M. H. Lu, L. Feng, and Y. F. Chen, New J. Phys. 17, 053016 (2015).

[21] Z.-G. Chen and Y. Wu, Phys. Rev. Appl. 5, 054021 (2016).

[22] R. Fleury, A. B. Khanikaev, and A. Alu, Nat. Commun. 7, 11744 (2016).
[23] C. He, Z. Li, X. Ni, X.-C. Sun, S.-Y. Yu, M.-H. Lu, X.-P. Liu, and Y.-F. Chen, Appl. Phys. Lett. 108, 031904 (2016).

[24] Q. Wei, Y. Tian, S. Y. Zuo, Y. Cheng, and X. J. Liu, Phys. Rev. B 95, 094305 (2017).

[25] Y.-G. Peng, C.-Z. Qin, D.-G. Zhao, Y.-X. Shen, X.-Y. Xu, M Bao, H. Jia, and X.-F. Zhu, Nat. Commun. 7, 13368 (2016).

[26] S. H. Mousavi, A. B. Khanikaev, and Z. Wang, Nat. Commun. 6, 8682 (2015).

[27] C. He, X. Ni, H. Ge, X.-C. Sun, Y.-B. Chen, M.-H. Lu, X.-P. Liu, and Y.-F. Chen, Nat. Phys. 12, 1124 (2016).

[28] Z. Zhang, Q. Wei, Y. Cheng, T. Zhang, D. Wu, and X. Liu, Phys. Rev. Lett. 118, 084303 (2017).

[29] J. Mei, Z. Chen, and Y. Wu, Sci. Rep. 6, 32752 (2016).

[30] J. Lu, C. Qiu, L. Ye, X. Fan, M. Ke, F. Zhang, and Z. Liu, Nat. Phys. 13, 369 (2017).

[31] B.-Z. Xia, T.-T. Liu, G.-L. Huang, H.-Q. Dai, J.-R. Jiao, X.-G. Zang, D.-J. Yu, S.-J. Zheng, and J. Liu, Phys. Rev. B 96, 094106 (2017).

[32] X. Wen, C. Qiu, J. Lu, H. He, M. Ke, and Z. Liu, J. Appl. Phys. 123, 091703 (2018).

[33] L. M. Nash, D. Kleckner, A. Read, V. Vitelli, A. M. Turner, and W. T. M. Irvine, Proc. Natl. Acad. Sci. U. S. A. 112, 14495 (2015).

[34] R. Suesstrunk and S. D. Huber, Science 349, 47 (2015).

[35] E. Prodan and C. Prodan, Phys. Rev. Lett. 103, 248101 (2009).

[36] P. Wang, L. Lu, and K. Bertoldi, Phys. Rev. Lett. 115, 104302 (2015).

[37] R. Fleury, D. L. Sounas, C. F. Sieck, M. R. Haberman, and A. Alù, Science 343, 516 (2014).

[38] See Supplemental Material at http://link.aps.org/supplemental/ 10.1103/PhysRevB.96.241306 for information about the pseudo-time-reversal symmetry, simulations, experiments, and comparison between topological edge states and transmission states. The dynamic views of intensity circulation around molecule center and the RCP/LCP chirality are shown in movie 1 . 
[39] J. Lu, C. Qiu, S. Xu, Y. Ye, M. Ke, and Z. Liu, Phys. Rev. B 89, 134302 (2014).

[40] J. Lu, C. Qiu, M. Ke, and Z. Liu, Phys. Rev. Lett. 116, 093901 (2016).

[41] M. Xiao, W.-J. Chen, W.-Y. He, and C. T. Chan, Nat. Phys. 11, 920 (2015).
[42] D. Torrent and J. Sánchez-Dehesa, Phys. Rev. Lett. 108, 174301 (2012).

[43] S.-Q. Shen, W.-Y. Shan, and H.-Z. Lu, Spin 01, 33 (2011).

[44] R. S. K. Mong and V. Shivamoggi, Phys. Rev. B 83, 125109 (2011). 